\title{
Performance Analysis of LTE system for 2x2 Rayleigh and Rician Fading Channel
}

\author{
Mithun Sah ${ }^{1}$, Achint Chugh ${ }^{2}$ \\ Mittal Institute of Technology ${ }^{1,2}$ \\ e-mail: smithun85@gmail.com ${ }^{1}$, achintchugh@gmail.com²
}

To cite this document:

Sah, M., \& Chugh, A. (2021). Performance Analysis of LTE system for $2 \times 2$ Rayleigh

and Rician Fading Channel. Aptisi Transactions on Technopreneurship (ATT), 3(1).

https://doi.org/10.34306/att.v3i1.131

\begin{abstract}
Development of LTE is a milestone in the field of communication engineering. Compared to the performance of $2 G$ the network involving $L T E$ provides much higher data rates and support much more services. The evolution to LTE has provided the users to utilize many commercial activities to bring forward to the public and provide them the improved services compared to older versions of communication technologies. As the growth of LTE has provided much faster network still there are improvements going on the new technology. Various performance measures have been taken into considerations has the networks has performed very well on such test. One of the performance analyses has been presented in this paper comparing the performance of the LTE system over the Rayleigh and Rician fading channel. In the paper the performance of the MIMO LTE has been analyzed for the networks with various modulation schemes for Rayleigh and Rician fading channel with a 2x2 MIMO channel for EPA OHz, EVA $5 \mathrm{~Hz}$, EVA $70 \mathrm{~Hz}$. By varying Signal to Noise Ratio (SNR), Bit Error Rate (BER) of LTE is calculated for different Multi-Input Multi-Output (MIMO) schemes. The analysis is concluded in terms of speed of the data rate in Mbps.
\end{abstract}

Keywords: Fourth Generation (4G), 3GPP (Third Generation Partnership Project), LTE (Long Term Evaluation), MIMO (Multiple Input Multiple Output), Evolved Node B (eNB), User

Equipment (UE).

\section{Introduction}

Wireless communication systems in modern days are highly emphasized on less power requirements, higher data rates, coverage enhancements and reliable communications. Multiple Input Multiple Output (MIMO) system can be identified as a contender for meeting such challenges. Higher spectral efficiency can be achieved by the use of MIMO channel technique and also it provides improved reliability for the ongoing communication environment [1][2]. An improvement in throughput and enhancement in coverage area for a communication system can then be achieved using cooperative relaying [3][4][5][6][7]. Using MIMO system, the need of high power at the transmitter end can be reduced which in turn reduces the co-channel and interchannel interference. It also ensures low interference with the other nodes of the communication system. Relaying protocols like amplify and forward (AF) and decode and forward (DF) are the two-technique utilized in the MIMO system. Using AF protocol, the relaying node forwards the received signal to the end receiver after amplification while using DF the received signal is forwarded to the destination after re-encoding and modulating the signal [8]. In both the AF and DF systems are implemented and are compared as per the implementation loss and the complexity in implementation. This also showed that the AF protocol is less complex and has low 
implementation loss i.e. The performance is very much similar to the theoretical values. Many performance studies on AF MIMO relaying shows that the instantaneous channel state information (CSI) of source-relay and relay-destination channels are available at the relaying node [9]. Moreover, adverse channel fading in wireless system can be overcome by the use of cooperative communication. In such type of fading scenario use of multiple relays helps to provide a reliable transmission from the source to the destination while using relay selection method these adverse channel effects can be mitigated. Relay selection plays a major role in reducing adverse channel effects, to increase the diversity order and to overcome the problem in half duplex loss [10][11]. The statistical approach for the instantaneous SNR at the receiver end is derived on the basis of probability density function (PDF), moments and cumulative distribution function (CDF). Long Term Evolution (LTE) standard is specified by the 3GPP wireless communication. This standard provides higher data rates, frequency flexibility, higher spectral efficiency and short round trip time [12]. This standard depends on Multiple Input and Multiple-Output (MIMO). Orthogonal Frequency. Division Multiplexing (OFDM), scheduling and link adaptation and on robust channel coding schemes [13]. LTE systems are compatible with widely used schemes like GPRS, HSPA, and WCDMA which helps mobile operators deploy LTE and provide faultless and speedy services to the customers [14]. Much modification has been done since LTE has been first presented to the customers. In its subsequent releases it has increased flexible configurations, lowered latencies and improved the data throughput. A peak data rate of 300 Mbps downlink and 75 Mbps uplink, bandwidth sized in $1.4,3,5,10,15$, or $20 \mathrm{MHz}$ and a radionetwork delay of less than $5 \mathrm{~ms}$ was introduced in its release R8. It also provided significant increase in spectrum efficiency compared to previous cellular systems like UMTS and HSPA. In its next release R9 it introduced network architecture improvements and some newer features like dual layer beam forming, location services and evolved multimedia broadcast and multicast services. LTE Advanced i.e., release 10 provided downlink data rates of $3 \mathrm{Gbps}$ and uplink data rates of $1.5 \mathrm{Gbps}$, higher antenna MIMO configurations supporting up to $4 \times 4$ uplinks and $8 \times 8$ downlinks along with Carrier Aggregation (CA). Enhancements to Carrier Aggregation, relay nodes and MIMO, coordinated multipoint transmission and reception and introduction of new frequency bands were included in release R11 of LTE systems. The LTE transmission and reception are based on OFDMA (Orthogonal Frequency Division Multiple Access) and SC-FDMA (Single Carrier Frequency Division Multiple Access). High PAPR is the drawback of the OFDMA scheme compared to SC FDMA. OFDMA scheme allocates users in time and frequency domain and using FFT it generates the transmitting signal. In OFDMA during MIMO transmission, optimum receivers can be implemented with allowable complexity since the wideband frequency selective channel is converted into many fading sub-channels. Due to low PAPR SCFDMA is more power efficient as compared to OFDMA which in turn enhances the power efficiency of the user equipment. In contrast to uplink, LTE output power vary depending on the frequency band used also the maximum Transmission power is $23 \mathrm{dBm}$ [15]. Packet Data Convergence Protocol (PDCP), Medium Access Control (MAC), The Physical layer (PHY) and Radio Link Control (RLC) are used in the LTE RAN (Radio Access Network). Using transport blocks which conveys data for at most two subframes the PHY transfers the data to and from the MAC layer. The simulation is carried out using the LTE system toolbox of MATLAB software. The work is performed in the PDSCH (Physical Downlink shared Channel) and PUSCH (Physical Uplink Shared Channel) [16]. The PHY layer carryout antenna mapping, the modulation and demodulation coding and decoding. Highly efficient means of conveying both data and control information between mobile UE (User Equipment) and enhanced base station (eNodeB) is the LTE physical layer [17]. This paper discusses the speed of the 2x2 MIMO systems in terms of Mbps. Like 2G or 3G LTE also has its own architecture. It is simply a network of Evolved Packet Core Network and base stations (eNB) as shown in figure below [18][19]. 


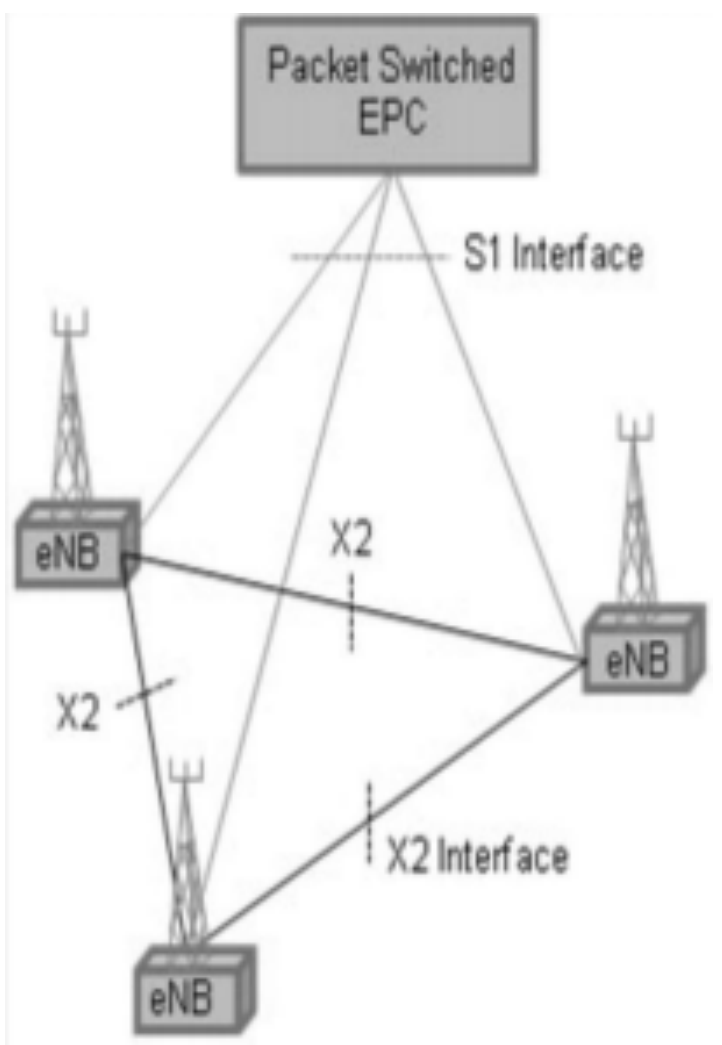

Figure.1: Architecture of LTE

In LTE no centralized intelligent controller exists, also the eNB systems are normally interconnected by a X2-interface and that of towards the core network via the S1-interface as shown in Figure.1. The simple reason of distribution of the systems in such a way among the base-stations in LTE is just to speed up the time required for connection set-up and to reduce the handover time [20][21][22].

\section{Research Method}

This architecture helps in the design of the LTE system for the transmission and reception of the data. The data is created randomly and is passed through various modules in the transmitter section to the fading channel and then to various block of receiver section. The Simulink model of the above-mentioned system is shown below:

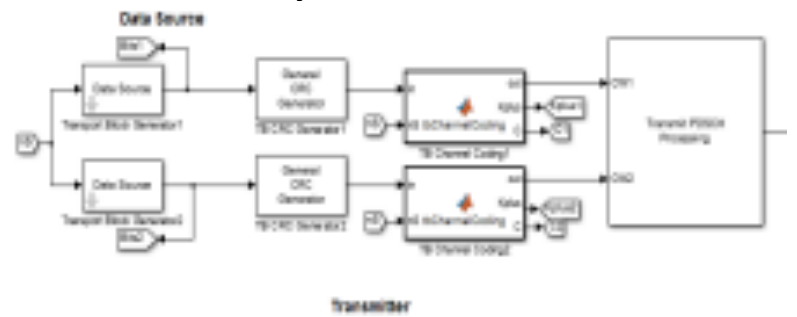

Figure.2: Transmitter Section 


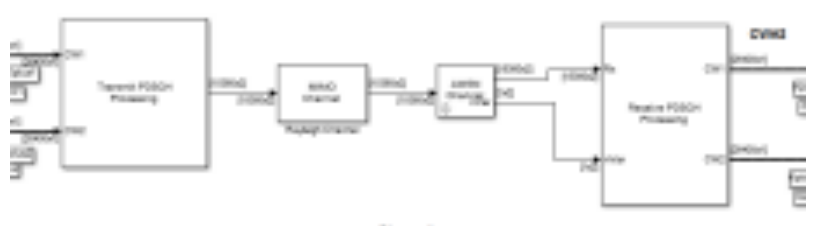

Figure.3: Rayleigh Fading Channel

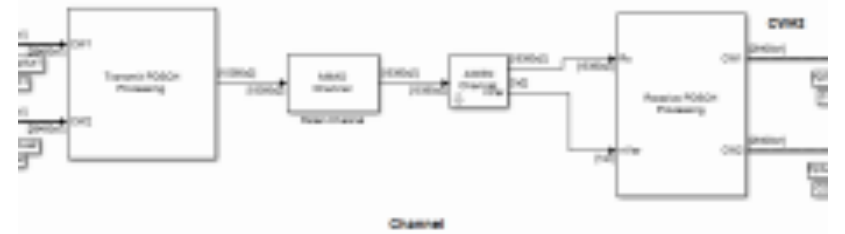

Figure.4: Rician Fading Channel

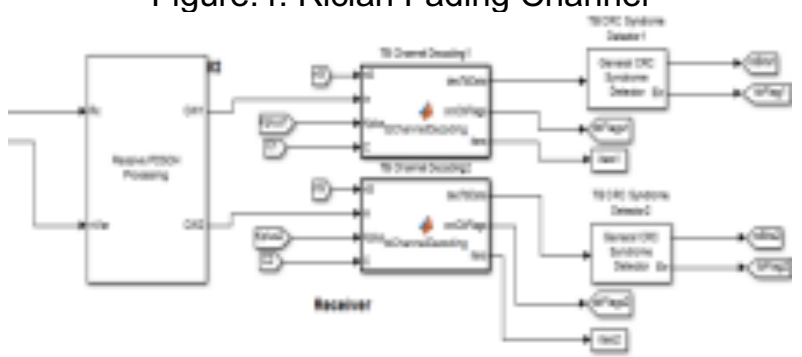

Figure.5: Receiver Section

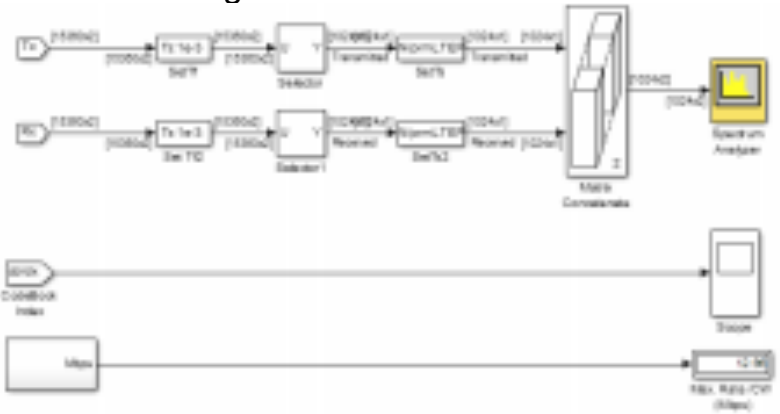

Figure.6: Measurements

The above figure explains the transmitter, receiver and measurement part of the simulation respectively. Transmitter section contains the data source generator, the CRC Generator, the Turbo Channel Coder and the Transmit PDSCH processing block. The MIMO Fading channel with AWGN is used as the channel to transmit the generated signal. At the receiver section, the Receive PDSCH processing block. Turbo Channel Decoder and the General CRC Syndrome Detector are present. The data source which is a random number generator is used to generate the data source which is to be transmitted to the receiver. The data source generated here is passed to the CRC Generator which adds the sufficient CRC bits to identify any error in the received bit while transmission. The Turbo channel coder codes the generated bits so as to effectively use the channel bandwidth which is then passed to the $\mathrm{PDSCH}$ processing block to transmit the data. The $\mathrm{PDSCH}$ processing block contains the PDSCH scrambler which is then passed to the modulator section. Till now the data sources are processed individually now the layer mapper performs the spatial multiplexing to make a single source then it is processed by the spatial multiplexing precoder then it is again remapped by resource element mapper to map it according to the resource grid chosen and then finally OFDM modulation is done for transmitting the processed data. This data or signal is then transmitted over the MIMO fading channel and AWGN Channel for modeling the channel transmission. At the receiver end firstly the Receive PDSCH process the received data which OFDM demodulates the received signal then the data is extracted from the signal which is passed to the MIMO receiver to demultiplex the data the layer demapper is employed [23][24]. The data 
obtained from the layer demapper is then passed to demodulator and then to the PDSCH descrambler.

The data obtained after descrambling are the received code words [25]. These codewords may or may not contain error which may occur at the channel section. At last, the error introduced by the fading AWGN channel is measured with the speed of transmission. In the measurement section a MATLAB function block is used to obtain the speed of the communication in terms of Mbps [26]. The system is configured as per the parameter shown below:

\begin{tabular}{|c|c|}
\hline PARAMETER & VALUE \\
\hline Channel Bandwidth & $5 \mathrm{MHz}$ \\
\hline Duplex mode & FDD \\
\hline Transmit Channel Modulation & OFDM \\
\hline Channel type & $\begin{array}{c}\text { Flat Static MIMO, EPA OHz, EVA } \\
5 \mathrm{~Hz}, \text { EVA 70Hz }\end{array}$ \\
\hline Fading Channel & Rayleigh / Rician \\
\hline FEC coding & Turbo coding \\
\hline SNR & $12.1 \mathrm{~dB}$ \\
\hline Modulation & QPSK, 16-QAM, \\
& $64-\mathrm{QAM}$ \\
\hline Subcarrier spacing & $15 \mathrm{KHz}$ \\
\hline Antenna diversity & $2 \times 2 \mathrm{MIMO}$ \\
\hline
\end{tabular}

Table 1: System Configuration

\section{Simulation Result and Analysis}

For the configured system as shown in Table 1, the performance is measured in terms of speed of the data transmission based on the acceptable SNR value given to the system [27][28]. The transmitted and received signal over the channel has been viewed in a spectrum analyzed as shown below: 


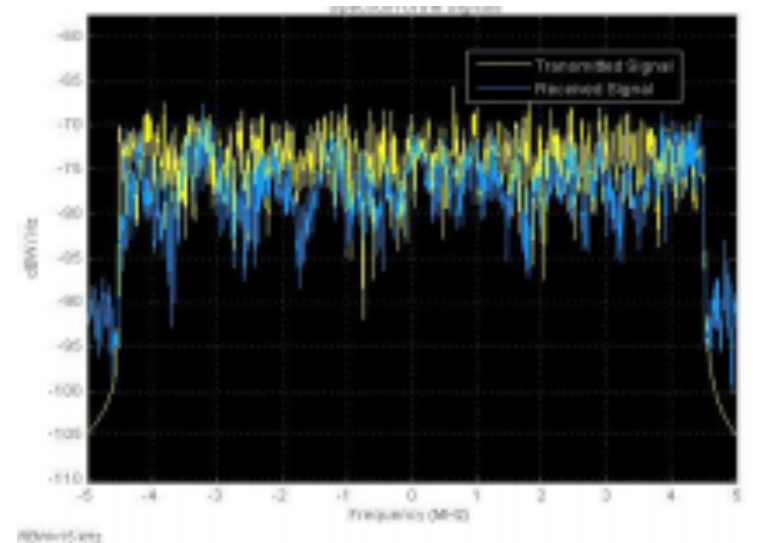

Figure.5: Transmit and received Signal spectrum for 16QAM, 2x2, EVA 5Hz channel with Rayleigh Fading Channel

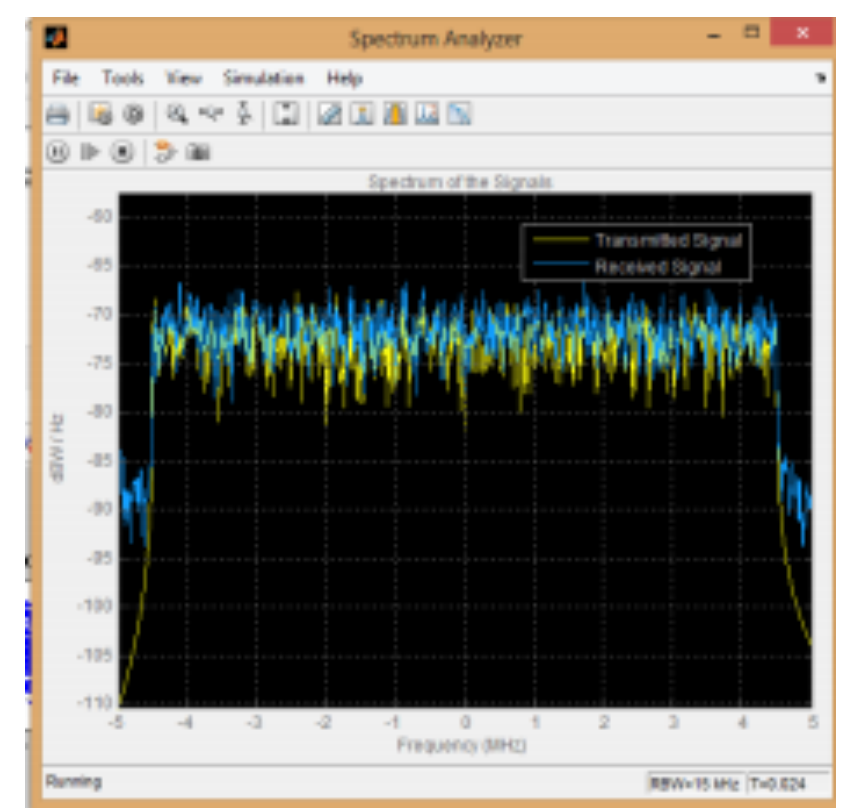

Figure.6: Transmit and received Signal spectrum for 16QAM, 2x2, EVA 5Hz channel with Rician Fading Channel

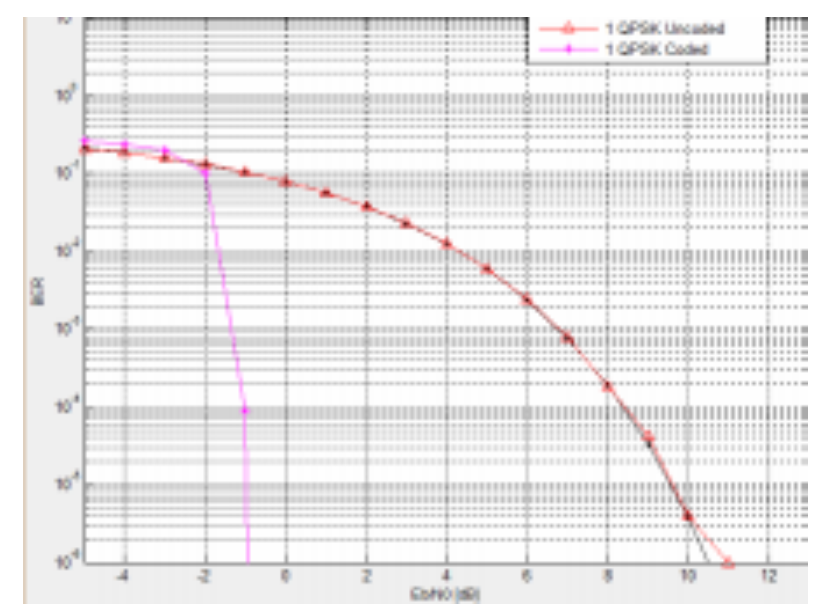

Figure.7: Bit Error Rate of the QPSK Signal 


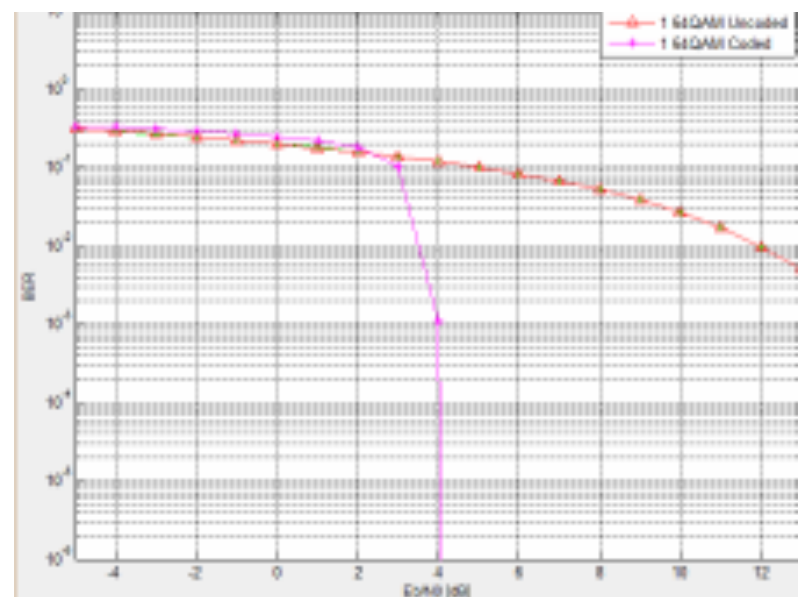

Figure.8: Bit Error Rate of the 64-QAM Signal

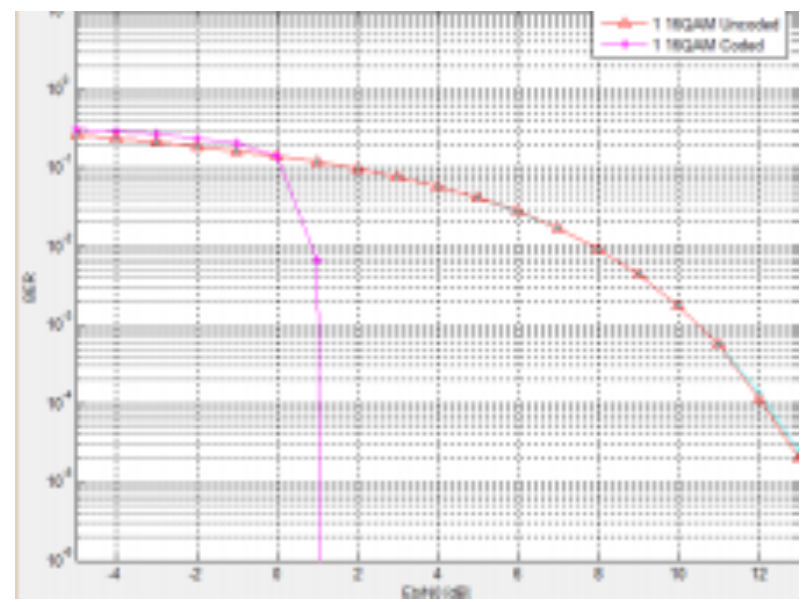

Figure.9: Bit Error Rate of the 16-QAM Signal
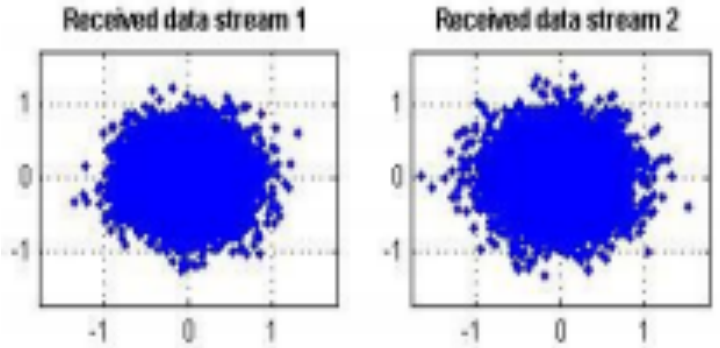

Figure.10: Received Data for 16QAM, 2x2, EVA 5Hz channel 

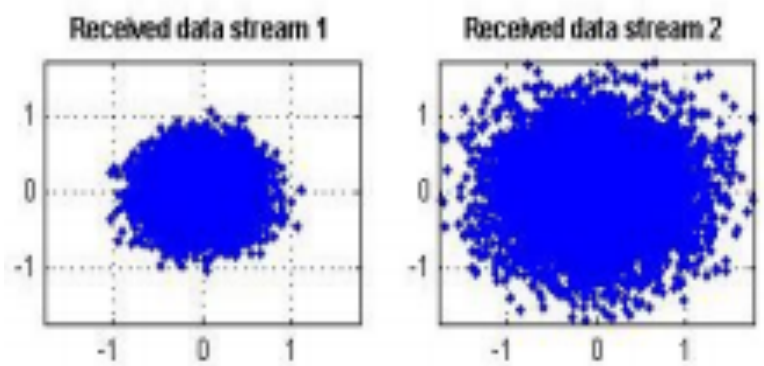

Figure.11: Received Data for 64QAM, 2X2, EPA OHZ channel

The speed analysis and Bit Error Rate for the configured system is shown in the table below:

\begin{tabular}{|c|c|c|c|}
\hline \multirow[t]{2}{*}{ Configuration } & \multirow[t]{2}{*}{$\begin{array}{l}\text { Speed in } \\
\text { Mbps }\end{array}$} & \multicolumn{2}{|c|}{$\begin{array}{l}\text { Bit Error Rate } \\
\text { (CW1, CW2) }\end{array}$} \\
\hline & & $\begin{array}{l}\text { Rayleigh } \\
\text { Fading } \\
\text { Channel }\end{array}$ & $\begin{array}{l}\text { Rician } \\
\text { Fading } \\
\text { Channel }\end{array}$ \\
\hline 16QAM,2x2, EPA OHz & 12.96 & $\begin{array}{l}0.1125 \\
0.0584\end{array}$ & $\begin{array}{l}0.1263 \\
0.4927\end{array}$ \\
\hline $\begin{array}{c}\text { 64QAM,2X2, EPA } \\
\text { OHZ }\end{array}$ & 19.6968 & $\begin{array}{l}0.2079 \\
0.1456\end{array}$ & $\begin{array}{l}0.2204 \\
0.4946\end{array}$ \\
\hline QPSK, 2x2, EPA OHz & 6.2 & $\begin{array}{l}0.0013 \\
0.0003\end{array}$ & $\begin{array}{l}0.0158 \\
0.4920\end{array}$ \\
\hline 16QAM,2x2, EVA 5Hz & 12.96 & $\begin{array}{l}0.1651 \\
0.1454\end{array}$ & $\begin{array}{l}0.1263 \\
0.4927\end{array}$ \\
\hline 64QAM,2x2, EVA 5Hz & 19.6968 & $\begin{array}{l}0.2513 \\
0.2343\end{array}$ & $\begin{array}{l}0.2204 \\
0.4946\end{array}$ \\
\hline QPSK, 2x2, EVA 5Hz & 6.2 & $\begin{array}{l}0.0400 \\
0.0228\end{array}$ & $\begin{array}{l}0.0158 \\
0.4920\end{array}$ \\
\hline 16QAM,2x2, EPA 5Hz & 12.96 & $\begin{array}{l}0.1977 \\
0.0700\end{array}$ & $\begin{array}{l}0.1263 \\
0.4927\end{array}$ \\
\hline 64QAM,2x2, EPA 5Hz & 19.6968 & $\begin{array}{l}0.2855 \\
0.1633\end{array}$ & $\begin{array}{l}0.2204 \\
0.4946\end{array}$ \\
\hline QPSK, 2x2, EPA 5Hz & 6.2 & $\begin{array}{l}0.0388 \\
0.0036\end{array}$ & $\begin{array}{l}0.0158 \\
0.4920\end{array}$ \\
\hline 16QAM,2x2, EVA $70 \mathrm{~Hz}$ & 12.96 & $\begin{array}{l}0.1300 \\
0.1326\end{array}$ & $\begin{array}{l}0.1263 \\
0.4927\end{array}$ \\
\hline 64QAM, 2x2, EVA70Hz & 19.6968 & $\begin{array}{l}0.2172 \\
0.2200\end{array}$ & $\begin{array}{l}0.2204 \\
0.4946\end{array}$ \\
\hline QPSK, 2x2, EVA $70 \mathrm{~Hz}$ & 6.2 & $\begin{array}{l}0.0310 \\
0.0321\end{array}$ & $\begin{array}{l}0.0158 \\
0.4920\end{array}$ \\
\hline
\end{tabular}




\begin{tabular}{|c|c|c|c|}
\hline 16QAM,2×2, flat static & 12.96 & $\begin{array}{l}0.2785, \\
0.0485\end{array}$ & $\begin{array}{l}0.1263, \\
0.4927\end{array}$ \\
\hline MIMO & & $\begin{array}{l}0.3597, \\
0.1463\end{array}$ & 0.2204, \\
64QAM, 2x2, flat static & \multirow{2}{*}{19.6968} & 0.4946 \\
\hline MIMO & & 0.0549, & 0.0158, \\
QPSK, 2x2, flat static & 6.2 & 0.0000 & 0.4920 \\
MIMO & & \multicolumn{2}{|l}{} \\
\hline
\end{tabular}

Above table shows speed of the transmission in Mbps and Bit Error Rate of the two code words considered for the transmission for 16QAM, 64QAM and QPSK Modulation for 2x2 MIMO fading channel of OFDM channel modulation. It has been observed that in case of Rician fading channel the BER is changing according to the modulation type used unlike the changes observed in case of Rayleigh fading channel which is changing according to the modulation type used as well as the channel type selected. It has also been observed that there is no change in the speed of the data transmission in Rayleigh or Rician fading channel used.

\section{Conclusion}

The result obtained from the LTE system shown in Figure.2 to Figure.7 is presented in Table 2. It is observed that for Rayleigh fading channel and a $2 \times 2$ MIMO system the speed of the system remains the same for all modulation types i.e., 16QAM, 64QAM and QPSK using OFDM at the transmitting section. However, the Bit Error Rate changes significantly of these modulation types. It is observed that for QPSK, $2 \times 2$. EPA OHz the Bit Error Rate is minimum for Code Word 1, while for Code Word 2 the minimum Bit Error Rate is obtained for QPSK, 2x2, flat static MIMO. It is also observed that the Bit Error Rate of QPSK, 2x2. EPA OHz is minimum for both the Code Words. So, it is inferred from this result that in case of QPSK for $2 \times 2$ MIMO channel the transmitting data undergoes minimum Bit Error. The BER is not affected much in case of Rician fading channel. Rather it is observed that in Rician fading channel the BER remains the same irrespective of channel type selected it only changes with respect to the modulation type used.

\section{References}

[1] S. M. Alamouti, "A simple transmit diversity technique for wireless communications," IEEE J. Sel. areas Commun., vol. 16, no. 8, pp. 1451-1458, 1998.

[2] G. J. Foschini and M. J. Gans, "On limits of wireless communications in a fading environment when using multiple antennas," Wirel. Pers. Commun., vol. 6, no. 3, pp. 311-335, 1998.

[3] A. Sendonaris, E. Erkip, and B. Aazhang, "User cooperation diversity. Part I. System description," IEEE Trans. Commun., vol. 51, no. 11, pp. 1927-1938, 2003.

[4] A. Sendonaris, E. Erkip, and B. Aazhang, "User cooperation diversity. Part II. Implementation aspects and performance analysis," IEEE Trans. Commun., vol. 51, no. 11, pp. 1939-1948, 2003.

[5] X. Zhang, W. Wang, and X. Ji, "Multiuser diversity in multiuser two-hop cooperative relay wireless networks: System model and performance analysis," IEEE Trans. Veh. Technol., vol. 58, no. 2, pp. 1031-1036, 2008.

[6] M. O. Hasna and M.-S. Alouini, "A performance study of dual-hop transmissions with fixed gain relays," IEEE Trans. Wirel. Commun., vol. 3, no. 6, pp. 1963-1968, 2004.

[7] A. Nosratinia, T. E. Hunter, and A. Hedayat, "Cooperative communication in wireless networks," IEEE Commun. Mag., vol. 42, no. 10, pp. 74-80, 2004.

[8] A. K. Pradhan, A. Routray, and A. Basak, "Power system frequency estimation using least mean square technique," IEEE Trans. power Deliv., vol. 20, no. 3, pp. 1812-1816, 2005.

[9] Y. Jing and H. Jafarkhani, "Single and multiple relay selection schemes and their achievable diversity orders," IEEE Trans. Wirel. Commun., vol. 8, no. 3, pp. 1414-1423, 2009.

[10] A. López, J.-C. Montaño, M. Castilla, J. Gutierrez, M. D. Borrás, and J. C. Bravo, "Power system frequency measurement under nonstationary situations," IEEE Trans. power Deliv., vol. 23, no. 2, pp. 562-567, 2008.

[11] J.-B. Kim and D. Kim, "Comparison of tightly power-constrained performances for opportunistic 
amplify-and-forward relaying with partial or full channel information," IEEE Commun. Lett., vol. 13, no. 2, pp. 100-102, 2009.

[12] T. Takpor and F. E. Idachaba, "Analysis and simulation of LTE downlink and uplink transceiver," 2014.

[13] X. Guo and P. Song, "Simulink based LTE system simulator." 2010.

[14] K. Zhou, J. Zhou, and Z. Xu, "Design of a high performance RF transceiver for TDD-LTE system," in 2012 IEEE/MTT-S International Microwave Symposium Digest, 2012, pp. 1-3.

[15] M. Suarez and O. Zlydareva, "LTE transceiver performance analysis in Uplink under various environmental conditions," in 2012 IV International Congress on Ultra Modern Telecommunications and Control Systems, 2012, pp. 84-88.

[16] N. Hou, K. Niu, Z. He, and S. Sun, "Test and performance analysis of PUSCH channel of LTE system," in 2013 5th IEEE International Symposium on Microwave, Antenna, Propagation and EMC Technologies for Wireless Communications, 2013, pp. 110-114.

[17] G. Indumathi and D. A. Joe, "Design of optimum physical layer architecture for a high data rate LTE uplink transceiver," in 2013 International Conference on Green High Performance Computing (ICGHPC), 2013, pp. 1-8.

[18] D. Astély, E. Dahlman, A. Furuskär, Y. Jading, M. Lindström, and S. Parkvall, "LTE: the evolution of mobile broadband," IEEE Commun. Mag., vol. 47, no. 4, pp. 44-51, 2009.

[19] M. A. Mohamed, H. M. Abd-ElAtty, M. E. A. A. El-Seoud, and W. M. Raslan, "Performance Analysis of LTE-Advanced Physical Layer," Int. J. Comput. Sci. Issues, vol. 11, no. 1, p. 80, 2014.

[20] E. U. T. R. Access, "User Equipment (UE) radio transmission and reception (Release 10)," 3GPP TS 36.101," V10, vol. 2, 2011.

[21] N. Magdalena, "3GPP a global initiative." 2013.

[22] P. SURYAWANSHI and P. MANOHAR, "Data Rate Analysis of LTE System for 2x2 MIMO Fading Channel," 2016.

[23] M. V. S. Lima, C. M. G. Gussen, B. N. Espíndola, T. N. Ferreira, W. A. Martins, and P. S. R. Diniz, "Open-source physical-layer simulator for LTE systems," in 2012 IEEE International Conference on Acoustics, Speech and Signal Processing (ICASSP), 2012, pp. 2781-2784.

[24] K. Arora, A. S. Bist, R. Prakash, and S. Chaurasia, "A Novel Approach for Facial Attendance: AttendXNet," Aptisi Trans. Technopreneursh., vol. 2, no. 2, pp. 104-111, 2020.

[25] K. Arora, A. S. Bist, R. Prakash, and S. Chaurasia, "Custom OCR for Identity Documents: OCRXNet," Aptisi Trans. Technopreneursh., vol. 2, no. 2, pp. 112-119, 2020.

[26] J. Hom, B. Anong, K. B. Rii, L. K. Choi, and K. Zelina, "The Octave Allegro Method in Risk Management Assessment of Educational Institutions," Aptisi Trans. Technopreneursh., vol. 2, no. 2, pp. 167-179, 2020.

[27] S. Khanna, "ICT Enabled Learning: A tool in Crisis Management," Aptisi Trans. Technopreneursh., vol. 2, no. 2, pp. 127-130, 2020.

[28] A. I. L. Wibowo, A. D. Putra, M. S. Dewi, and D. O. Radianto, "Study of Divergence of Go Public Company's Financial Performance Based on Website Before and After Merger Using Window Period Method TIME Frame 2015-2017," Aptisi Trans. Technopreneursh., vol. 1, no. 1, pp. $27-$ 51, 2019. 